\title{
On Near-Wall Collateral Flow in Skewed Turbulent Boundary Layers
}

\author{
K. S. Hebbar* \\ National Aeronautical Laboratory, Bangalore, India
}

\section{Introduction}

NE of the unresolved questions in skewed turbulent boundary layers concerns the existence of a collateral mean flowfield very close to the wall. Most of the existing data in the inner region of the boundary layer (particularly in the region extending up to $y^{+} \approx 50$ ) seem to have been somewhat restricted by the size and/or response of the probes used, resulting in inadequate spatial resolution. ${ }^{1-3}$ A careful examination of various experimental polar plots (see, for example, Ref. 4, Chap. 7) indicates near-wall collateral flow, i.e., in the inner region very close to the wall (sometimes extending up to local-to-freestream velocity ratios of as high as 0.5 ) the mean velocity vector does not change its direction. It should be noted that in most of these plots, the inner region between the wall and the apex was constructed by drawing a mean line through the origin and a few available data points (as low as two in some cases) near the apex. ${ }^{1}$ However, recent measurements of Rogers and Head ${ }^{5}$ using a specially designed hot-wire anemometer device showed skewed flow much closer to the wall; the data point closest to the wall corresponding to a resultant velocity ratio (local to freestream) of about 0.2 . Similar trends are also observed in the later data of Vermeulen. ${ }^{6}$ It is clear, therefore, that more (reliable) data are still needed to resolve experimentally the question of the existence or nonexistence of a near-wall collateral flowfield.

Received Feb. 22, 1977; revision received May 16, 1977. Index category: Boundary Layers and Convective Heat TransferTurbulent.

-Scientist, Aerodynamics Division. Member AIAA. 
This is all the more important because the existence of such a collateral flowfield is not predicted either by numerical calculations or by a sublayer analysis of the flowfield. In fact, numerical calculations by East and Pierce ${ }^{\mathrm{I}}$ indicate that the assumptions of near-wall collateral flow, as suggested by many experimentalists, may not be correct. ${ }^{2}$ A careful study of the boundary-layer equations in the neighborhood of the wall leads to the following relation (see Ref. 4, p. 102):

$$
\frac{\partial^{2} W}{\partial U^{2}}=\frac{\mu}{\tau_{w x}^{2}}\left[\frac{\partial p}{\partial z}-\frac{\tau_{w z}}{\tau_{w x}} \cdot \frac{\partial p}{\partial x}\right]
$$

In a collateral flowfield $(\partial W / \partial U)=$ constant, i.e., $\left(\partial^{2} W / \partial U^{2}\right)=0$.

Thus, Eq. (1) implies a collateral flowfield only when either the resultant pressure gradient is zero or it is in the direction of the resultant wall shear stress. Therefore, in general, it implies a noncollateral flowfield.

The question of the existence of a near-wall collateral flow is of special interest in the context of defining a limiting wall streamline direction to numerical difference solutions, which make use of a rate equation for modeling turbulent shear stress. ${ }^{2}$ This Note is intended to address this question in the light of some new data extremely close to the wall (much closer than has been reported hitherto) obtained in a recent experimental investigation. ${ }^{7,8}$

\section{Experiments and Results}

An experimental investigation ${ }^{7.8}$ was conducted to study the near-wall region of a three-dimensional turbulent air boundary layer relaxing in a nominally zero external pressure gradient behind a transverse hump (in the form of a $30 \mathrm{deg}$ swept, 5-ft chord wing-type model) faired into the side wall of a low-speed wind tunnel. Measurements were made at selected port locations behind the trailing edge with a traverse mechanism (designed to minimize probe interference) that was externally mounted on the test wall. These included nearwall measurements with a single rotated hot-wire probe and wall shear stress measurements with a flush mounted hot-film gage, a sublayer fence, and two Preston probes. All measurements were made at an upstream Reynolds number of $3.25 \times 10^{5}$ per foot, with local freestream velocities of 53-57 fps. The boundary layer (approximately 3.5 in. thick near the trailing edge) had a maximum crossflow velocity ratio of 0.145 and a maximum crossflow angle of $21.9 \mathrm{deg}$ close to the wall.

Figure 1 shows the mean direction profiles in the inner layer obtained from hot-wire surveys. Figure 2 represents some typical polar plots of mean velocity profile. The resultant friction velocity $\vec{U}^{*}$ used in evaluating the wall coordinates was based on the resultant skin friction $\bar{c}_{f}$, determined from the 0.032-in-diam Preston probe measurement at the respective port location. The closest distance probed was 0.0005 in. from the wall and the estimated lowest $y^{+}$values were less than unity. With wall proximity corrections ${ }^{7}$ for hot-wire readings, the resultant velocity ratios (corrected local resultant mean velocity) closest to the wall were as low as 0.01 .

\section{Discussion}

The experimental mean direction profiles exhibit the usual features characteristic of a simple crossflow profile, but with a relatively smaller collateral region adjacent to the wall. With the exception of the profiles at the last three survey stations (ports 5,6, and 7), which are practically two dimensional, the data indicate the existence of a collateral flowfield up to $y^{+}=9.7$ to $17.6 ; y^{+}$increasing with $\vec{U}^{*}$. The value of $y^{+}$at which the maximum crossflow angle occurs varies from 27.1 to $144.8, y^{+}$increasing with $\bar{U}^{*}$ as before. The most striking feature in these profiles is the presence of a narrow region of slightly decreasing crossflow angle (1 deg or less) that extends from the point of maximum crossflow angle down to the outer limit of the collateral region.

While doing the hot wire surveys, first a continuous increase in the crossflow angle was registered as the survey progressed toward the wall, but the angle started decreasing slowly after the point of maximum crossflow angle. This decreasing trend (observed consistently during the hot-wire surveys at all port locations) continued in a narrow region close to the wall until, finally, the measured crossflow angle remained constant in a small region immediately adjacent to the wall. The consistency of the data at several locations suggests good repeatability.

It is suspected that the presence of a slight lateral adverse pressure gradient opposing the crossflow in the wall region ${ }^{7}$ caused a small collateral layer followed by a small decrease of crossflow angle close to the wall. It is appropriate to remark here that in the extreme case of reversed lateral pressure gradient impressed on a skewed boundary layer, the flow reversal should begin at the wall and proceed outward to the boundary-layer edge, leading to simultaneous lateral skewing. ${ }^{9}$

An approximate but simple analysis of the sublayer flowfield based on Eq. (1) and measured local wall pressure gradients did indeed predict the unusual behavior of decreasing crossflow angle. Details of this analysis may be found in Ref. 7. The crossflow profiles at ports 1 and 2 which had appreciable wall crossflow angles were selected for the

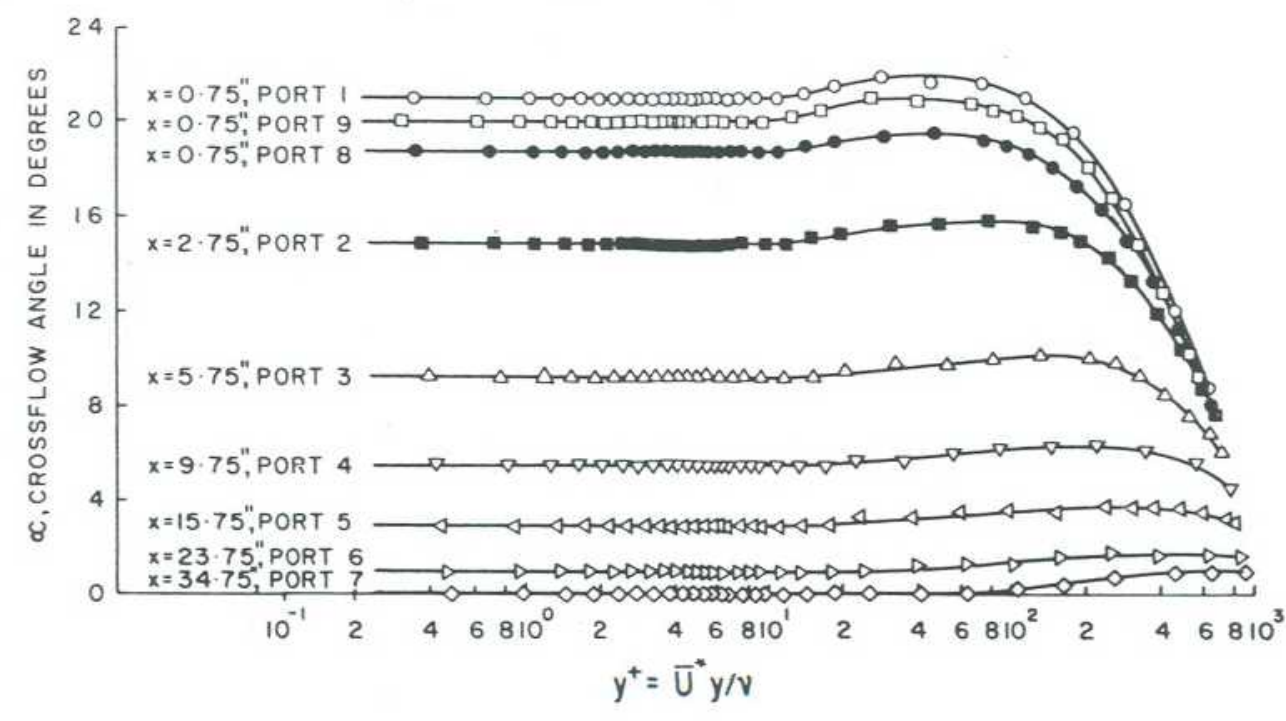

Fig. 1 Mean direction profiles (crossflow angles) in the inner layer of the relaxing boundary layer. 
Fig. 2 Polar plots of mean velocity profile (ports 1,8, and 9).

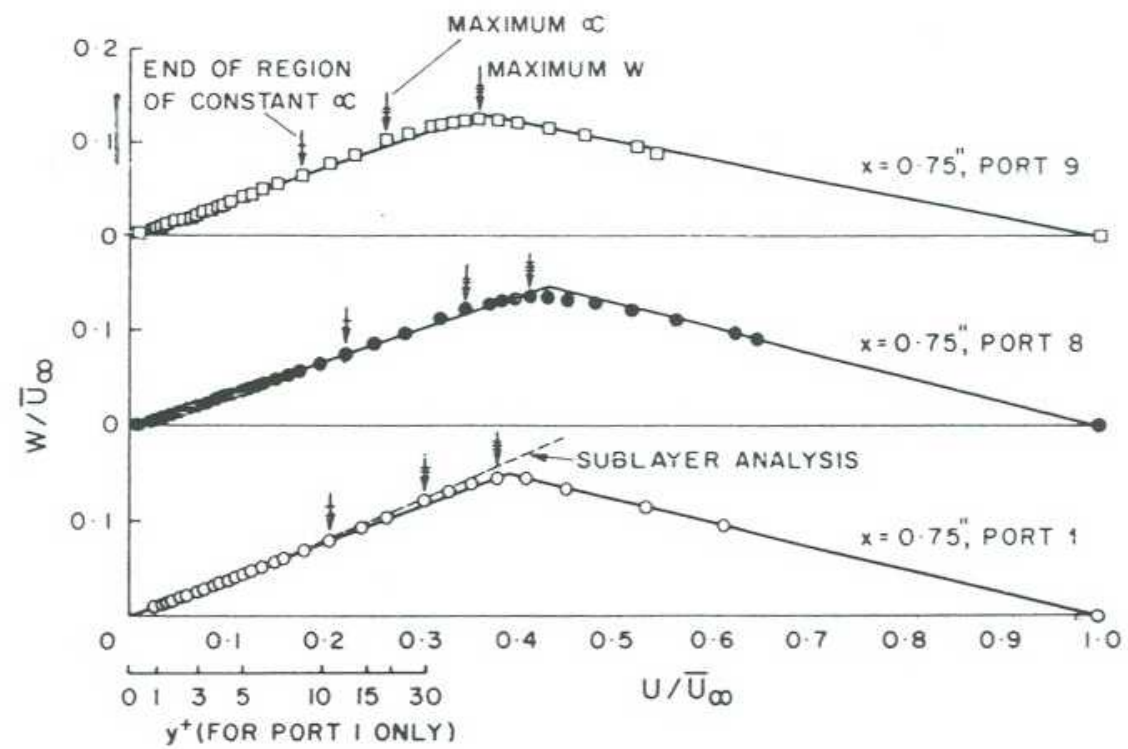

analysis because the estimates of the wall pressure gradients at these locations were considered to be more accurate and reliable. Some typical values of change of crossflow angle $\Delta \alpha$ predicted by the analysis are compared with the experimental data in Refs. 7 and 8 . The analysis predicts conservative results (overestimates), the overestimation increasing with distance from the wall. This is to be expected as the effect of the wall static pressure field decreases with distance from the wall. The overestimation is also seen in the predicted profile shapes which have a slight curvature upward (Fig. 2). Within the limitations of the analysis, the estimated decrease of crossflow angle compares favorably with the measured value.

\section{Conclusion}

This Note clearly points to the importance of and difficulties in obtaining reliable measurements and reliable flow conditions near the wall of skewed flows. It suggests that even slight lateral adverse pressure gradients could be initiating reversal of the crossflow, with the first sign of this reversal being the collateral layer and decreasing flow angles near the wall. With these considerations of the near-wall flowfield, it is conceivable that in the absence of the small local transverse pressure gradients close to the wall, the skewing of the flow could have been much more pronounced practically down to the wall (limited only by the resolution of the sensor), implying a noncollateral flowfield consistent with the equations of motion in the neighborhood of the wall.

\section{Acknowledgment}

This work formed a part of the investigation carried out by the author at the Aerospace Engineering Department of the University of Maryland. The author wishes to thank W.L. Melnik and A.E. Winkelmann.

\section{References}

${ }^{1}$ East, J.L., Jr. and Pierce, F.J., "Explicit Numerical Solution of the Three-Dimensional Incompressible Turbulent Boundary Layer," AIAA Journal, Vol. 10, Sept. 1972, pp. 1216-1223.

${ }^{2}$ Pierce, F.J. and East, J.L., Jr., "Near-Wall Collateral Flow in Three-Dimensional Boundary Layers," AIAA Journal, Vol. 10, March 1972, pp. 334-336.

${ }^{3}$ Pierce, F.J. and Zimmerman, B.B., "Wall Shear Stress Inference from Two- and Three-Dimensional Turbulent Boundary Layer Velocity Profiles," Journal of Fluids Engineering, Transactions of ASME, Ser. 1, Vol. 95, 1973, pp. 61-67.

${ }^{4}$ Nash, J.F. and Patel, V.C., Three-Dimensional Turbulent Boundary Layers, SBC Technical Books, Atlanta, Ga., 1972.

${ }^{3}$ Rogers, B.K. and Head, M.R., "Measurement of ThreeDimensional Boundary Layers," The Aeronautical Journal of the Royal Aeronautical Society, Vol. 73,1969, pp. $796-798$.
${ }^{6}$ Vermeulen, A.J., "Measurements of Three-Dimensional Turbulent Boundary Layers," Ph.D. Dissertation, Univ. of Cambridge, 1971.

${ }^{7}$ Hebbar, K.S., "An Experimental Investigation of the Near-Wall Region of a Three-Dimensional Incompressible Turbulent Boundary Layer Relaxing in a Zero Pressure Gradient," Ph.D. Dissertation, Univ. of Maryland, 1976.

${ }^{8}$ Hebbar, K.S. and Melnik, W.L.. "Measurements in the NearWall Region of a Relaxing Three-Dimensional Low Speed Turbulent Air Boundary Layer," Dept. of Aerospace Engineering, Univ. of Maryland, TR No. AE-76-1, July 1976.

${ }^{9}$ Klinksiek, W.F. and Pierce, F.J., "Simultaneous Lateral Skewing in a Three-Dimensional Turbulent Boundary Layer Flow," Journal of Basic Engineering, Transactions of ASME, Ser. D, Vol. 92, 1970, pp. 83-92.

\section{Ice-Crystal/Shock-Layer Interaction in Hypersonic Flight}

\author{
T. C. Lin* and N. A. Thyson $\dagger$ \\ Avco Systems Division, Wilmington, Mass.
}

W HEN a re-entry vehicle flying through the Earth's atmosphere traverses a cloud, raindrops or ice crystals may strike the nosetip at high speed, causing serious damage to the heatshield. The shock layer surrounding the vehicle tends to shatter, decelerate, and melt the incoming debris and hence acts as a shield against the erosive environment. In order to make accurate predictions of the nosetip erosion, one must first understand the kinematics of these ambient particles and their thermal environment. The analytical results presented here are focused on the dynamics of ice crystals in the shock layer, although the formulation can be extended to particles other than ice.

\section{Heating Environment}

Previous investigations concerning the kinematics of particles in the shock layer include either vaporization or melting processes. ${ }^{1,2}$ In the latter case, it is assumed that the

Received March 15, 1977; revision received July 12, 1977.

Index categories: Multiphase Flows; Supersonic and Hypersonic Flow.

*Presently Technical Staff Member, The Aerospace Corporation. Member AIAA.

tDepartment Head. Member AIAA. 